\title{
The auditory and non-auditory brain areas involved in tinnitus. An emergent property of multiple parallel overlapping subnetworks
}

\section{Sven Vanneste * and Dirk De Ridder}

Brai $^{2} n$, TRI and Department of Neurosurgery, University Hospital Antwerp, Antwerp, Belgium

\author{
Edited by: \\ Jos J. Eggermont, University of \\ Calgary, Canada \\ Reviewed by: \\ Dave Langers, University Medical \\ Center Groningen, Netherlands \\ Winfried Schlee, Ulm University, \\ Germany

\section{*Correspondence:} \\ Sven Vanneste, Brai ${ }^{2} n, T R I$ and \\ Department of Neurosurgery, \\ University Hospital Antwerp, \\ Wilrijkstraat 10, 2650 Edegem, \\ Belgium. \\ e-mail:sven.vanneste@ua.ac.be
}

Tinnitus is the perception of a sound in the absence of an external sound source. It is characterized by sensory components such as the perceived loudness, the lateralization, the tinnitus type (pure tone, noise-like) and associated emotional components, such as distress and mood changes. Source localization of quantitative electroencephalography ( $\mathrm{qEEG}$ ) data demonstrate the involvement of auditory brain areas as well as several non-auditory brain areas such as the anterior cingulate cortex (dorsal and subgenual), auditory cortex (primary and secondary), dorsal lateral prefrontal cortex, insula, supplementary motor area, orbitofrontal cortex (including the inferior frontal gyrus), parahippocampus, posterior cingulate cortex and the precuneus, in different aspects of tinnitus. Explaining these non-auditory brain areas as constituents of separable subnetworks, each reflecting a specific aspect of the tinnitus percept increases the explanatory power of the non-auditory brain areas involvement in tinnitus. Thus, the unified percept of tinnitus can be considered an emergent property of multiple parallel dynamically changing and partially overlapping subnetworks, each with a specific spontaneous oscillatory pattern and functional connectivity signature.

Keywords: EEG, non-auditory brain areas, emergent property, multiple parallel overlapping subnetworks

\section{INTRODUCTION}

Consciousness is a crucial aspect of being human. One specific component of consciousness is the conscious perception of auditory stimuli. Hearing is a crucial sensory domain that helps to localize as well as recognize a sound source and is essential for communication. Our auditory function helps us to understand the world, maintain social contacts and to detect dangerous situations. In humans and other vertebrates, hearing is performed primarily by the auditory system. Vibrations are detected by the ear and translated into nerve impulses that are processed by the auditory cortex. Recent research has shown, however, that activity in the primary auditory cortex is necessary, but not sufficient condition for an auditory stimulus to gain access to consciousness (Boly et al., 2004). It has recently become clear that in order to perceive an auditory percept, hierarchically higher-order multimodal association areas are required (Boly et al., 2004; Laureys, 2005), similarly to what has been proposed in the visual (Dehaene et al., 2006) and somatosensory system (Laureys et al., 2002; Boly et al., 2005).

Understanding the brain mechanisms involved in the simplest forms of auditory conscious perception, such as noise and tones from the environment (i.e., externally generated) is a crucial start for gaining knowledge about auditory consciousness specifically and consciousness at large. However, a sound can also be internally generated. That is, perceiving a sound in the absence of an external sound source. This phenomenon is also known as tinnitus. In most cases this phantom sound resolves spontaneously within seconds or minutes. However, tinnitus persists in $5-10 \%$ of the population in western countries (Heller, 2003; Eggermont and Roberts, 2004), and interferes severely with the quality of life in $5-26 \%$ within this tinnitus population (Axelsson and Ringdahl, 1989; Heller, 2003). Moreover, the prevalence of chronic tinnitus increases with age, peaking at $14.3 \%$ in people between 60 and 69 years of age (Shargorodsky et al., 2010).

Phenomenologically, tinnitus can be perceived unilaterally or bilaterally and characterized as a pure tone, a narrow band noise or polyphonic. Tinnitus is usually evaluated both for its perceived loudness and annoyance or distress level. Yet, not everyone who experiences tinnitus becomes chronically distressed and measures of tinnitus loudness rarely correlate with experienced distress (Andersson and Westin, 2008). Distress can play an important part in the development of tinnitus, as distress might act as a potential trigger for sudden hearing loss and onset of tinnitus, but is not a necessity (Schmitt et al., 2000). Distress might unfavorably influence habituation via hyperarousal processes, but is not a requirement (Hallam, 1996). Tinnitus symptoms themselves can act as a stressor resulting in higher physiological arousal and psychological distress, but this is not always the case (Alpini and Cesarani, 2006).

In this review we try to map and disentangle the different brain areas generating an auditory phantom percept. We will only focus on simple auditory phantom percepts such as tones and noise and not on more complex sounds such as hearing voices or music. Although very common, tinnitus is not well understood. Clinical data indicate the involvement of peripheral auditory structures in tinnitus (Nicolas-Puel et al., 2002). This is suggested by the 
fact that tinnitus is often related to damage of the cochlea or the auditory nerve such as in presbyacusis, noise induced hearing loss, drug-related hearing loss, Meniere's disease, or other inner ear pathologies (Lockwood et al., 2002). Furthermore it has been demonstrated that psychoacoustic characteristics of tinnitus like pitch overlap with the frequency spectrum of an individual's hearing loss (Norena et al., 2002; Norena and Eggermont, 2003, 2006). In addition in animal models it was revealed that a peripheral mechanism involving the N-methyl-D-aspartate (NMDA) glutamatergic receptors in the cochlea can be generator of tinnitus (Guitton et al., 2003). On the other hand, an increasing amount of data shows the role played by activation and remodeling of various central cortical or subcortical structures to cause or to perpetuate tinnitus symptomatology (Muhlnickel et al., 1998; Eggermont and Roberts, 2004; Eggermont, 2005; Weisz et al., 2005). Investigating the neurophysiological differences in the characteristics of tinnitus perception could lead to a better understanding of pathological auditory neural activity. Therefore, we first discuss the different auditory and non-auditory brain areas involved in tinnitus and their potential function within the tinnitus network. Secondly, we try to combine these different brain areas involved in tinnitus in a multiple brain subnetworks.

\section{THE AUDITORY AND NON-AUDITORY BRAIN AREAS INVOLVED IN TINNITUS}

Based on previous quantitative electroencephalography ( $\mathrm{qEEG}$ ) research the following areas have been implicated in tinnitus: the auditory cortex, the subgenual and dorsal anterior cingulate cortex, the dorsolateral prefrontal cortex, the insula, the supplementary motor area, the orbitofrontal cortex (including the inferior frontal gyrus), the posterior cingulate cortex, the precuneus and the parahippocampus. Table 1 and Figure 1 give an overview of the different brain areas obtained based on qEEG research in tinnitus and their involvement in specific tinnitus characteristics.

\section{THE AUDITORY CORTEX}

Animal experiments have demonstrated that the degree of behavioral importance of an external sound is related to the representational expansion of its frequency in the primary auditory cortex (Rutkowski and Weinberger, 2005), and that the auditory cortex is involved in tinnitus (Engineer et al., 2011). But also in humans it was shown that the auditory cortex plays a role in tinnitus (van der Loo et al., 2009). In comparison to a control group both left and right-sided tinnitus patients had an increased gamma band activity in both the left and right primary and secondary auditory cortex (Vanneste et al., 2011a). This is the reason why primary and secondary auditory cortices are considered as important potential targets for the treatment of tinnitus (De Ridder et al., 2006a, 2007a,b). The rationale is that this phantom sound might be related to an increased neuronal activity within the auditory cortex secondary to the imbalance between excitatory and inhibitory mechanisms or an adjustment of auditory gain mechanisms (Norena, 2011). The difference could be triggered by altered auditory inputs which may support functional reorganization in synaptic connections. Neural hyperactivity has been found in subcortical structures (cochlear nuclei, inferior colliculi, medial geniculate bodies) and auditory cortical regions (primary and secondary auditory cortex) in animal models of tinnitus and hearing loss (Jastreboff and Sasaki, 1986; Jastreboff, 1990; Brozoski et al., 2002).

Based on MEG data, thalamocortical dysrhythmia has been proposed as a pathophysiological model for the development of gamma band activity related to the tinnitus percept (Llinás et al., 1999). According to this model tinnitus is caused by an abnormal, spontaneous, and constant gamma band activity $(>30 \mathrm{~Hz})$ generated as a consequence of hyperpolarization of specific thalamic nuclei, in casu the medial geniculate body. In normal circumstances auditory stimuli increase thalamocortical rhythms to gamma band activity (Joliot et al., 1994). In the deafferented state, however, oscillatory activity decreases from resting state alpha activity $(8-12 \mathrm{~Hz})$ to theta band activity $(4-7 \mathrm{~Hz})$ (Steriade, 2006). As a result, lateral inhibition is reduced inducing a surrounding gamma band activity known as the "edge effect" (Llinás et al., 1999, 2005). Lorenz et al. (Lorenz et al., 2009) reported an inverse relationship between alpha and gamma activity over subjects calculated for sources seeded in auditory regions. The inverse relationship was presented for tinnitus and control tinnitus group. Synchronized gamma band activity in the auditory cortex is proposed to bind auditory events into one coherent conscious auditory percept (Ribary et al., 1991; Tiitinen et al., 1993; Joliot et al., 1994; Llinas et al., 1994, 1998; Crone et al., 2001). In addition it was found that tinnitus perceived loudness is correlated to increased contralateral gamma band activity in the auditory cortex indicating that gamma band activity is important in tinnitus (van der Loo et al., 2009).

\section{THE PARAHIPPOCAMPUS}

The differences between uni- and bilateral tinnitus are reflected by high frequency EEG activity (i.e., beta and gamma) in the parahippocampus (Vanneste et al., 2011c). That is, unilateral tinnitus patients showed increased high frequency activity in the right parahippocampal area. This same brain area is also involved at an alpha rhythm in patients with a high distress and in noncoping with tinnitus. In addition, based on a region of interest analysis, whether tinnitus is perceived on the left side or right side tinnitus is dependent on gamma-band activity of the contralateral parahippocampal area (Vanneste et al., 2011c). In contrast to expectation, for the auditory cortex no differences were found between left-sided and right-sided tinnitus patients. In addition, narrow band noise tinnitus patients have increased activity in the parahippocampal area in comparison to pure tone tinnitus patients at the gamma frequency band (Vanneste et al., 2010a).

The involvement of the parahippocampus in tinnitus might be related to the constant updating of the tinnitus percept from memory thereby preventing habituation (De Ridder et al., 2006b). The posterior parahippocampal area is involved in auditory habituation as demonstrated by electrophysiological studies of auditory sensory gating both in animals (Bickford et al., 1993) and humans implanted with electrodes in the parahippocampus and hippocampus for epilepsy monitoring (Boutros et al., 2008). The hippocampal involvement in tinnitus pathophysiology is also demonstrated by histopathological findings of posterior hippocampus lesions in patients, who experience 
Table 1 | Overview results based on resting-state EEG in tinnitus patients.

\begin{tabular}{|c|c|c|c|c|}
\hline Brain region & BA & Function & Frequency band & Ref \\
\hline & BA22 & Tinnitus with recent onset $<$ Chronic tinnitus & gamma & Vanneste et al., 2011b \\
\hline & $\mathrm{BA} 41$ & Positive correlation with tinnitus intensity & gamma & van der Loo et al., 2009 \\
\hline \multirow[t]{6}{*}{ Parahippocampus } & BA36 & Low distress < High distress & alpha1, alpha2 & Vanneste et al., 2010b \\
\hline & & Left-sided tinnitus < Right-sided Tinnitus (left) & gamma & Vanneste et al., 2011a \\
\hline & & Control group $<$ Left and Right-sided tinnitus & gamma & Vanneste et al., 2011a \\
\hline & & Pure tone $<$ Narrow band noise tinnitus & gamma & Vanneste et al., 2010a \\
\hline & & Control group $<$ Narrow band noise tinnitus & beta3, gamma & Vanneste et al., 2011c \\
\hline & & Unilateral tinnitus $<$ Bilateral tinnitus & & \\
\hline & & Correlation with distress & alpha, beta & De Ridder et al., 2011b \\
\hline \multirow[t]{3}{*}{ Subgenual } & BA25 & Low distress $<$ High distress & alpha1, alpha2 & Vanneste et al., 2010b \\
\hline & & Coping with tinnitus $<$ Non-coping & alpha1, alpha2 & Vanneste et al., 2010b \\
\hline & & Correlation with TQ & alpha, beta & De Ridder et al., 2011b \\
\hline \multirow[t]{2}{*}{ DLPFC } & BA9 & Low distress coping $>$ High distress coping & alpha1, alpha2 & Vanneste et al., 2010b \\
\hline & BA46 & & & \\
\hline \multirow[t]{4}{*}{ Insula } & BA13 & Tinnitus with recent onset $<$ Chronic tinnitus & beta3 & Vanneste et al., 2011b \\
\hline & & Coping with tinnitus $<$ Non-coping with tinnitus & alpha1, alpha2 & Vanneste et al., 2010b \\
\hline & & Correlation with TQ and activity (left) & theta, alpha, gamma & van der Loo et al., 2011 \\
\hline & & Correlation between TQ and activity (right) & delta, gamma & van der Loo et al., 2011 \\
\hline & BA47 & Control group $<$ Bilateral tinnitus & beta3 & Vanneste et al., 2011c \\
\hline \multirow[t]{4}{*}{ Posterior cingulate cortex } & BA23 & Low distress $>$ High distress & alpha2 & Vanneste et al., 2010b \\
\hline & & Pure tone $<$ Narrow band noise tinnitus & beta3 & Vanneste et al., 2010a \\
\hline & & Control group $<$ Narrow band noise tinnitus & beta3 & Vanneste et al., 2010a \\
\hline & & Control group Pure tone tinnitus & beta3, gamma & Vanneste et al., 2010a \\
\hline \multirow[t]{3}{*}{ Precuneus } & BA7 & Low distress $>$ High distress & alpha2 & Vanneste et al., 2010b \\
\hline & & Coping with tinnitus $>$ Non-coping with tinnitus & alpha1, alpha2 & Vanneste et al., 2010b \\
\hline & & Low distress coping tinnitus $<$ High distress coping & alpha2 & Vanneste et al., 2010b \\
\hline
\end{tabular}

$D L P F C$, dorsal lateral prefrontal cortex; Ref, reference.

tinnitus as a symptom of methyltin intoxications (Rey et al., 1984; Kreyberg et al., 1992). Furthermore, supraselective amytal injection in the anterior choroidal artery that supplies the amygdalohippocampal area is capable to suppress the pure tone component of tinnitus transiently by suppressing local activity (De Ridder et al., 2006b). The parahippocampal area together with the posterior cingulate cortex activity might be load dependent, as noise-like tinnitus constitutes multiple frequencies in contrast to pure tone tinnitus (Vanneste et al., 2010a). Hence, it has been proposed that a fundamental function of the (para)hippocampal structures is the establishment of auditory memory for tinnitus (Shulman, 1995).

\section{THE DORSAL ANTERIOR CINGULATE CORTEX}

A recent study, using source localization in EEG, revealed that distress in tinnitus patients is related to increased beta activity in the dorsal part of the anterior cingulate cortex and the amount of distress correlates with an alpha activity in several brain areas such as the amygdala, anterior cingulate cortex, insula, and parahippocampus (Vanneste et al., 2010b). A comparison 


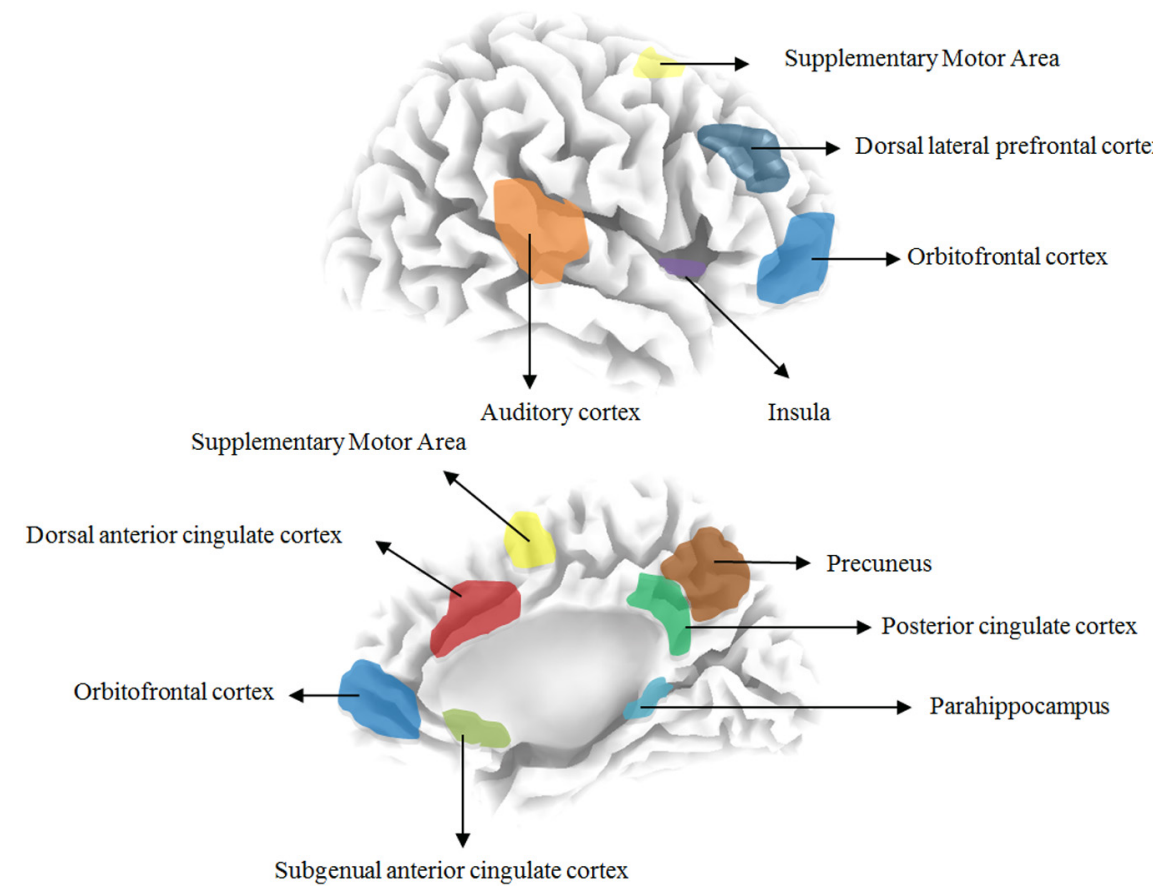

FIGURE 1 | Overview the different brain areas based on resting state EEG in tinnitus patients.

between recent onset and chronic tinnitus is related to differential activity and connectivity in a network comprising the auditory cortices, insula, dorsal anterior cingulate cortex and premotor cortex. Based on a blind source separation technique, tinnitus can be characterized by at least four independent components, two of which are posterior cingulate based, one based on the subgenual anterior cingulate and one based on the parahippocampus (De Ridder et al., 2011b). Only the subgenual component correlates with distress. When compared to a normative sample, group independent components analysis reveals that distress is characterized by two anterior cingulate based components. Spectral analysis of these components demonstrates that distress in tinnitus is related to alpha and beta changes in a network consisting of the subgenual anterior cingulate cortex extending to the pregenual and dorsal anterior cingulate cortex as well as the ventromedial prefrontal cortex/orbitofrontal cortex, insula, and parahippocampus. This network overlaps partially with brain areas implicated in distress in patients suffering from pain, functional somatic syndromes and, posttraumatic stress disorder, and might, therefore, represents an aspecific distress network. The dorsal part of the anterior cingulate cortex is one of the possible generators of frontal midline theta (Asada et al., 1999). Furthermore, it has been established that frontal midline theta oscillations are involved in attentional processes (Inanaga, 1998), and that both sympathetic and parasympathetic indices are increased during the appearance of frontal midline theta (Kubota et al., 2001). Whenever new information is presented, activity levels of the dorsal anterior cingulate cortex reflect the salience of the new information for predicting future outcomes (Critchley, 2005; Behrens et al., 2007), guiding optimal decision-making in an uncertain world (Kennerley et al., 2006). The human dorsal anterior cingulate cortex has developed a parallel specialization for motivational drive via a thalamocortical pathway relaying in the mediodorsal thalamus (Craig, 2002). Thus, the dorsal anterior cingulate might be involved in persisting attention to the tinnitus (Vanneste et al., 2010b; De Ridder et al., 2011b).

\section{THE SUBGENUAL ANTERIOR CINGULATE CORTEX}

The subgenual anterior cingulate cortex extending into nucleus accumbens-ventral tegmental area is involved in processing of aversive sounds (Zald and Pardo, 2002) and unpleasant music (Blood et al., 1999) as well as tinnitus (Muhlau et al., 2006). It has been implicated as the key component of social distress (Masten et al., 2009). This area in animals has been considered a visceromotor cortex, due to its connections with the parasympathetic nucleus tractus solitaries (Frysztak and Neafsey, 1994) and the sympathetic areas in the periaquaductal gray (Ongur and Price, 2000). Furthermore, it is functionally connected to the amygdala, insula, parahippocampus, orbitofrontal cortex, and ventrolateral prefrontal cortex and anticorrelated to the dorsal anterior cingulate cortex and precuneus. As such the subgenual anterior cingulate cortex could be important as an emotional component for tinnitus.

\section{THE DORSOLATERAL PREFRONTAL CORTEX}

Recently the dorsolateral prefrontal cortex has been associated with tinnitus-related distress (Vanneste et al., 2010b). It is known that the dorsal lateral prefrontal cortex has a bilateral facilitatory effect on auditory memory storage (Alain et al., 1998) and contains auditory memory cells (Bodner et al., 1996). The dorsal lateral prefrontal cortex also exerts early inhibitory modulation of input to primary auditory cortex in humans (Knight et al., 
1989) and has been found to be associated with auditory attention (Alain et al., 1998; Lewis et al., 2000; Voisin et al., 2006) resulting in top-down modulation of auditory processing (Mitchell et al., 2005). This was further confirmed by electrophysiological data indicating that tinnitus might occur as the result of a dysfunction in the top-down inhibitory processes (Norena et al., 1999). Interestingly, a recent study reported that coupling between dorsal anterior cingulate cortex and the right frontal lobe correlates negatively with tinnitus intrusiveness, which is defined by the authors as how bothersome and obtrusive the tinnitus is perceived (Schlee et al., 2008). However, in the above mentioned study it is not specified which part of the anterior cingulate cortex and prefrontal cortex is involved. Additionally, Jastreboff described the prefrontal cortex as a "candidate for the integration of sensory and emotional aspects of tinnitus" (Jastreboff, 1990). This is in accordance with the idea that the dorsal lateral prefrontal cortex in general could be considered as an area involved in the integration of emotion and cognition (Gray et al., 2002). Nevertheless, further research is needed to clarify the role of the dorsolateral prefrontal cortex in tinnitus.

\section{INSULA}

It was shown that the tinnitus questionnaire (TQ) scores are correlated to heart rate rariability markers, and related to neural activity in left and right anterior insula (van der Loo et al., 2011). It was shown that tinnitus distress is related to sympathetic activation, in part mediated via the right anterior insula. In addition the insula is activated in non-coping tinnitus at the alpha frequency band. The left insula is correlated with the TQ at theta, alpha, and gamma frequency band, while the right insula is correlated with delta and gamma frequency band.

The function of the dorsal anterior cingulate cortex and insula might be to integrate motivationally important information with appropriate bodily responses (Critchley et al., 2001) related to the survival needs of the body (Craig, 2003). In addition the insula together with the dorsal anterior cingulate cortex have also been referred to as the salience network (Seeley et al., 2007). This network has been implicated in bottom-up detection of salient events and coordinating appropriate responses (Medford and Critchley, 2010; Menon and Uddin, 2010). Activity in this network is correlated with improved sound detection thresholds, showing a role in the direction of attentional resources toward audition (Sadaghiani et al., 2009). The activation of the salience network suggests that the brain allocates an importance to auditory stimulus and might as such also signify importance to the internally generated tinnitus sound. Activation of the insula and dorsal anterior cingulate cortex during a phantom percept might be considered maladaptive. Imaging studies on the insula associated this area with subjective emotional and bodily awareness (Craig, 2003), as well as interoception (Craig, 2003). The anterior insula has been implicated in autonomic nervous system control (Oppenheimer et al., 1992; Oppenheimer, 1993; Critchley et al., 2004; Critchley, 2005) and might, therefore, be related to the autonomic components involved in distress (Critchley et al., 2000; Wang et al., 2005), induced by the phantom sound. Tinnitus distress is indeed correlated to sympathetic activation, in part mediated via the right anterior insula (van der Loo et al., 2011). Furthermore alpha activity in both the left and right anterior insula was also found for patients with severe tinnitus-related distress who can or cannot cope with these phantom sounds (Vanneste et al., 2010b). Although, the insula seems like an important brain area involved in tinnitus, further research is needed to elucidate what the exact role is of the insula in tinnitus.

\section{THE SUPPLEMENTARY MOTOR AREA}

For a sensory stimulus to be consciously perceived, activation of the early sensory areas is a prerequisite but not sufficient (Boly et al., 2005; Dehaene et al., 2006). The (visual) global workspace model suggests conscious perception of sensory events requires sensory cortex activation embedded in a cortical network, the global workspace, extending beyond the primary sensory regions including prefrontal, parietal, and cingulate cortices. Similarly, auditory stimuli need activation of the primary auditory cortex to be consciously perceived. However, this is not sufficient (Laureys et al., 2000; Boly et al., 2005). Studies performed on patients in vegetative state who do not have conscious auditory percepts reveal that auditory stimuli still activate the primary auditory cortex but that there is no functional connectivity to frontal areas in these patients. Primary auditory cortex activation might be only related to loudness coding (Jancke et al., 1998) and not the percept per se, similarly to what has been demonstrated at a single-cell level for somatosensory stimuli in the primary somatosensory cortex: stimulus intensity is encoded in the primary somatosensory cortex, while the conscious percept seems to be located in the frontal cortex, more precisely within the supplementary motor area (de Lafuente and Romo, 2005). In addition, Melloni et al., found that theta oscillations in the frontal regions including the supplementary motor area are essential for conscious perception during maintenance interval of visual stimuli (Melloni et al., 2007). Taking these findings together, it can be hypothesized that synchronized gamma activity in the auditory cortex is responsible for the tinnitus loudness (van der Loo et al., 2009), while synchronized theta activity in the supplementary motor area might be accountable for part of the conscious perception of the phantom sound, similar to the conscious perception for somatosensory stimuli.

\section{THE ORBITOFRONTAL CORTEX (INCLUDING THE INFERIOR FRONTAL GYRUS)}

Previous research has already shown that orbitofrontal cortex is important for emotional processing of sounds (Wheeler et al., 1993; Damasio, 1996; Dias et al., 1996; Blood et al., 1999). For example, it was revealed that patients with orbitofrontal cortex lesions had reduced self-evaluated perception of the unpleasantness of the acoustic probe stimulus (Angrilli et al., 2008). The orbitofrontal cortex has connections with other limbic areas important for processing of emotion (Beauregard, 2007). Female tinnitus patients have been found to be more emotionally responsive to tinnitus-related distress (Dineen et al., 1997). They also differ in physiological responses to negative emotional stimuli in comparison to males (Bradley et al., 2001; Gard and Kring, 2007). Koch et al. found that an interaction between negative emotion and working memory in females involved activation of the 
orbitofrontal cortex, suggesting that during the cognitive control of emotion, females mainly recruit the emotion-associated areas (Koch et al., 2007).

The orbitofrontal cortex together with the insula plays a key role in the top-down modulation of automatic or peripheral physiological responses to emotional experiences (Craig, 2003; Phillips et al., 2003; Critchley et al., 2004; Ohira et al., 2006). More synchronized connectivity between the orbitofrontal cortex and the insula is seen in tinnitus for females. It can, therefore, be hypothesized that the orbitofrontal cortex becomes recruited more often for female tinnitus patients in order to modulate the autonomic physiological responses evoked by tinnitus.

\section{THE POSTERIOR CINGULATE CORTEX AND PRECUNEUS}

qEEG data indicate that the posterior cingulate cortex is important in both pure tone and narrow band noise tinnitus (Vanneste et al., 2010a) as well as tinnitus-related distress (Vanneste et al., 2011c). In addition, the precuneus is active within the alpha frequency in patients who can cope with their tinnitus and have a low distress (Vanneste et al., 2011c). Together with the parahippocampal area, activation in the posterior cingulate cortex and precuneus has been associated with the brain's "default" network (Raichle et al., 2001). These regions deactivate when people engage in controlled processing and thought processes. According to this account, default activity is an inverse function of the task demand, where higher demands reduce activity in the default network because mental resources are used to perform a task (Gusnard et al., 2001; McKiernan et al., 2006). As the parahippocampal area as well as the posterior cingulate cortex and precuneus become more active, instead of becoming deactivated during the tinnitus perception, one can hypothesize the tinnitus generators might become integrated in the default mode in tinnitus patients. In addition, the precuneus area is a highly integrative structure, supposed to be involved in visuo-spatial imagery, episodic memory, self-consciousness, and the shifting of attention (Le et al., 1998). The precuneus is also involved in unpleasant music perception (Blood et al., 1999), auditory imagery (Yoo et al., 2001), and auditory memory retrieval (Buckner et al., 1996).

\section{REFERENCES}

Alain, C., Woods, D. L., and Knight, R. T. (1998). A distributed cortical network for auditory sensory memory in humans. Brain Res. 812, 23-37.

Alpini, D., and Cesarani, A. (2006). Tinnitus as an alarm bell: stress reaction tinnitus model. ORL J. Otorhinolaryngol. Relat. Spec. 68 , 31-36. discussion 36-37.

Andersson, G., and Westin, V. (2008). Understanding tinnitus distress: introducing the concepts of moderators and mediators. Int. J. Audiol. (Suppl. 2), S106-S111.

Angrilli, A., Bianchin, M., Radaelli, S., Bertagnoni, G., and Pertile, M. (2008). Reduced startle reflex and aversive noise perception in

\section{MULTIPLE PARALLEL DYNAMICALLY CHANGING AND PARTIALLY OVERLAPPING SUBNETWORKS}

Taking the results in previous sections together the same brain areas occur in the different analyses for tinnitus that are related to different acoustic characteristics such as the tonal nature, lateralization, loudness level, tinnitus duration as well as for the affective components such as distress and mood changes. As such, tinnitus can be seen as the consequence of multiple brain subnetworks involved in the different aspects of tinnitus, both acoustic and affective. Thus, the unified percept of tinnitus, as perceived by the patient, e.g., a loud distressing left-sided pure tone tinnitus, might be considered as an emergent property of multiple parallel dynamically changing and partially overlapping subnetworks, each with a specific spontaneous oscillatory pattern signature. This interpretation casts doubts concerning the sole participation of only one critical circuit in phantom perception. Phantom percepts result from auditory deafferentation and reach awareness only when increased neuronal activity in the primary auditory cortex is connected to a larger network involving frontal and parietal areas (De Ridder et al., 2011a). It is possible that different brain subnetworks overlap and might all be involved in how a patient perceives his/her tinnitus.

\section{CONCLUSION}

Source localization of $\mathrm{qEEG}$ data demonstrate the involvement of auditory brain areas as well as several non-auditory brain areas such as the anterior cingulate cortex (dorsal and subgenual), auditory cortex (primary and secondary), dorsal lateral prefrontal cortex, insula, supplementary motor area, orbitofrontal cortex (including the inferior frontal gyrus), parahippocampus, posterior cingulate cortex and the precuneus, in different aspects of tinnitus. However, few conceptual explanations have been given for all these regions. Evaluating these areas as parts of separable subnetworks, each network representing a specific clinical aspect of tinnitus might help to explain their involvement in tinnitus. Thus, the unified percept of tinnitus can be considered an emergent property of multiple parallel dynamically changing and partially overlapping subnetworks, each with a specific spontaneous oscillatory pattern and functional connectivity signature.

Behrens, T. E., Woolrich, M. W. Walton, M. E., and Rushworth, M. F. (2007). Learning the value of information in an uncertain world. Nat. Neurosci. 10, 1214-1221.

Bickford, P. C., Luntz-Leybman, V., and Freedman, R. (1993). Auditory sensory gating in the rat hippocampus: modulation by brainstem activity. Brain Res. 607, 33-38.

Blood, A. J., Zatorre, R. J., Bermudez, P. and Evans, A. C. (1999). Emotional responses to pleasant and unpleasant music correlate with activity in paralimbic brain regions. Nat. Neurosci. 2, 382-387.

Bodner, M., Kroger, J., and Fuster, J. M. (1996). Auditory memory cells in dorsolateral prefrontal cortex. Neuroreport 7, 1905-1908.
Boly, M., Faymonville, M. E., Peigneux, P., Lambermont, B., Damas, P., Del Fiore, G., Degueldre, C., Franck, G., Luxen, A., Lamy, M., Moonen, G., Maquet, P., and Laureys, S. (2004). Auditory processing in severely brain injured patients: differences between the minimally conscious state and the persistent vegetative state. Arch. Neurol. 61, 233-238.

Boly, M., Faymonville, M. E., Peigneux, P., Lambermont, B., Damas, F., Luxen, A., Lamy, M., Moonen, G., Maquet, P., and Laureys, S. (2005). Cerebral processing of auditory and noxious stimuli in severely brain injured patients: differences between VS and MCS. Neuropsychol. Rehabil. 15, 283-289. 
Boutros, N. N., Mears, R., Pflieger, M. E., Moxon, K. A., Ludowig, E., and Rosburg, T. (2008). Sensory gating in the human hippocampal and rhinal regions: regional differences. Hippocampus 18, 310-316.

Bradley, M. M., Codispoti, M., Sabatinelli, D., and Lang, P. J. (2001). Emotion and motivation II: sex differences in picture processing. Emotion 1, 300-319.

Brozoski, T. J., Bauer, C. A., and Caspary, D. M. (2002). Elevated fusiform cell activity in the dorsal cochlear nucleus of chinchillas with psychophysical evidence of tinnitus. J. Neurosci. 22, 2383-2390.

Buckner, R. L., Raichle, M. E., Miezin, F. M., and Petersen, S. E. (1996). Functional anatomic studies of memory retrieval for auditory words and visual pictures. J. Neurosci. 16, 6219-6235.

Craig, A. D. (2002). How do you feel? Interoception: the sense of the physiological condition of the body. Nat. Rev. Neurosci. 3, 655-666.

Craig, A. D. (2003). Interoception: the sense of the physiological condition of the body. Curr. Opin. Neurobiol. $13,500-505$.

Critchley, H. D. (2005). Neural mechanisms of autonomic, affective, and cognitive integration. J. Comp. Neurol. 493, 154-166.

Critchley, H. D., Corfield, D. R., Chandler, M. P., Mathias, C. J., and Dolan, R. J. (2000). Cerebral correlates of autonomic cardiovascular arousal: a functional neuroimaging investigation in humans. J. Physiol. 523(Pt 1), 259-270.

Critchley, H. D., Mathias, C. J., and Dolan, R. J. (2001). Neural activity in the human brain relating to uncertainty and arousal during anticipation. Neuron 29, 537-545.

Critchley, H. D., Wiens, S., Rotshtein, P., Ohman, A., and Dolan, R. J. (2004). Neural systems supporting interoceptive awareness. Nat. Neurosci. 7, 189-195.

Crone, N. E., Boatman, D., Gordon, B., and Hao, L. (2001). Induced electrocorticographic gamma activity during auditory perception. Brazier Award-winning article, 2001. Clin. Neurophysiol. 112, 565-582.

Damasio, A. R. (1996). The somatic marker hypothesis and the possible functions of the prefrontal cortex. Philos. Trans. R. Soc. Lond. B Biol. Sci. 351, 1413-1420.

Dehaene, S., Changeux, J. P., Naccache, L., Sackur, J., and Sergent, C.
(2006). Conscious, preconscious, and subliminal processing: a testable taxonomy. Trends Cogn. Sci. 10, 204-211.

de Lafuente, V., and Romo, R. (2005). Neuronal correlates of subjective sensory experience. Nat. Neurosci. 8 , 1698-1703.

De Ridder, D., de Mulder, G., Verstraeten, E., Seidman, M., Elisevich, K., Sunaert, S., Kovacs, S., K. van der Kelen, van de Heyning, P., and Moller, A. (2007a). Auditory cortex stimulation for tinnitus. Acta Neurochir. Suppl. 97, 451-462.

De Ridder, D., de Mulder, G., Menovsky, T., Sunaert, S., and Kovacs, S. (2007b). Electrical stimulation of auditory and somatosensory cortices for treatment of tinnitus and pain. Prog. Brain Res. 166, 377-388.

De Ridder, D., de Mulder, G., Verstraeten, E., van der Kelen, K., Sunaert, S., Smits, M., Kovacs, S., Verlooy, J., van de Heyning, P., and Moller, A. R. (2006a). Primary and secondary auditory cortex stimulation for intractable tinnitus. ORL J. Otorhinolaryngol. Relat. Spec. 68, 48-54. discussion 54-55.

De Ridder, D., Fransen, H., Francois, O., Sunaert, S., Kovacs, S., and van de Heyning, P. (2006b). Amygdalohippocampal involvement in tinnitus and auditory memory. Acta Otolaryngol. Suppl. 556, 50-53.

De Ridder, D., Elgoyhen, A. B., Romo, R., and Langguth, B. (2011a). Phantom percepts: tinnitus and pain as persisting aversive memory networks. Proc. Natl. Acad. Sci. U.S.A. $108,8075-8080$.

De Ridder, D., Vanneste, S., and Congedo, M. (2011b). The distressed brain: a group blind source separation analysis on tinnitus. PLoS One 6:e24273. doi: 10.1371/journal.pone.0024273

Dias, R., Robbins, T. W., and Roberts, A. C. (1996). Dissociation in prefrontal cortex of affective and attentional shifts. Nature 380 , 69-72.

Dineen, R., Doyle, J., and Bench, J. (1997). Audiological and psychological characteristics of a group of tinnitus sufferers, prior to tinnitus management training. Br. J. Audiol. $31,27-38$.

Eggermont, J. J. (2005). Tinnitus: neurobiological substrates. Drug Discov. Today 10, 1283-1290.

Eggermont, J. J., and Roberts, L. E. (2004). The neuroscience of tinnitus. Trends Neurosci. 27, 676-682.
Engineer, N. D., Riley, J. R., Seale, J. D., Vrana, W. A., Shetake, J. A., Sudanagunta, S. P., Borland, M. S., and Kilgard, M. P. (2011) Reversing pathological neural activity using targeted plasticity. Nature 470, 101-104.

Frysztak, R. J., and Neafsey, E. J. (1994). The effect of medial frontal cortex lesions on cardiovascular conditioned emotional responses in the rat. Brain Res. 643 181-193.

Gard, M. G., and Kring, A. M. (2007). Sex differences in the time course of emotion. Emotion 7, 429-437.

Gray, J. R., Braver, T. S., and Raichle, M. E. (2002). Integration of emotion and cognition in the lateral prefrontal cortex. Proc. Natl. Acad. Sci. U.S.A. 99, 4115-4120.

Guitton, M. J., Caston, J., Ruel, J., Johnson, R. M., Pujol, R., and Puel, J. L. (2003). Salicylate induces tinnitus through activation of cochlear NMDA receptors. J. Neurosci. 23, 3944-3952.

Gusnard, D. A., Akbudak, E., Shulman, G. L., and Raichle, M. E. (2001) Medial prefrontal cortex and selfreferential mental activity: relation to a default mode of brain function. Proc. Natl. Acad. Sci. U.S.A. 98 4259-4264

Hallam, R. S. (1996). Correlates of sleep disturbance in chronic distressing tinnitus. Scand. Audiol. 25, 263-266.

Heller, A. J. (2003). Classification and epidemiology of tinnitus. Otolaryngol. Clin. North Am. 36 239-248.

Inanaga, K. (1998). Frontal midline theta rhythm and mental activity. Psychiatry Clin. Neurosci. 52, 555-566.

Jancke, L., Shah, N. J., Posse, S., GrosseRyuken, M., and Muller-Gartner H. W. (1998). Intensity coding of auditory stimuli: an fMRI study. Neuropsychologia 36, 875-883.

Jastreboff, P. J. (1990). Phantom auditory perception (tinnitus): mechanisms of generation and perception. Neurosci. Res. 8, 221-254.

Jastreboff, P. J., and Sasaki, C. T. (1986). Salicylate-induced changes in spontaneous activity of single units in the inferior colliculus of the guinea pig. J. Acoust. Soc. Am. 80 1384-1391.

Joliot, M., Ribary, U., and Llinas, R. (1994). Human oscillatory brain activity near $40 \mathrm{~Hz}$ coexists with cognitive temporal binding. Proc. Natl. Acad. Sci. U.S.A. 91, 11748-11751.

Kennerley, S. W., Walton, M. E., Behrens, T. E., Buckley, M. J., and Rushworth, M. F. (2006). Optimal decision making and the anterior cingulate cortex. Nat. Neurosci. 9, 940-947.

Knight, R. T., Scabini, D., and Woods, D. L. (1989). Prefrontal cortex gating of auditory transmission in humans. Brain Res. 504, 338-342.

Koch, K., Pauly, K., Kellermann, T. Seiferth, N. Y., Reske, M., Backes, V., Stocker, T., Shah, N. J., Amunts, K. Kircher, T., Schneider, F., and Habel, U. (2007). Gender differences in the cognitive control of emotion: an fMRI study. Neuropsychologia 45 , 2744-2754.

Kreyberg, S., Torvik, A., Bjorneboe, A., Wiik-Larsen, W., and Jacobsen, D. (1992). Trimethyltin poisoning: report of a case with postmortem examination. Clin. Neuropathol. 11, 256-259.

Kubota, Y., Sato, W., Toichi, M., Murai, T., Okada, T., Hayashi, A., and Sengoku, A. (2001). Frontal midline theta rhythm is correlated with cardiac autonomic activities during the performance of an attention demanding meditation procedure. Brain Res. Cogn. Brain Res. 11, 281-287.

Laureys, S. (2005). The neural correlate of (un)awareness: lessons from the vegetative state. Trends Cogn. Sci. 9, 556-559.

Laureys, S., Faymonville, M. E., Degueldre, C., Fiore, G. D., Damas, P., Lambermont, B., Janssens, N., Aerts, J., Franck, G., Luxen, A., Moonen, G., Lamy, M., and Maquet, P. (2000). Auditory processing in the vegetative state. Brain 123(Pt 8), 1589-1601.

Laureys, S., Faymonville, M. E., Peigneux, P., Damas, P., Lambermont, B., Del Fiore, G., Degueldre, C., Aerts, J., Luxen, A., Franck, G., Lamy, M., Moonen, G., and Maquet, P. (2002). Cortical processing of noxious somatosensory stimuli in the persistent vegetative state. Neuroimage 17 , 732-741.

Le, T. H., Pardo, J. V., and Hu, X. (1998). 4 T-fMRI study of nonspatial shifting of selective attention: cerebellar and parietal contributions. J. Neurophysiol. 79, 1535-1548.

Lewis, J. W., Beauchamp, M. S., and deYoe, E. A. (2000). A comparison of visual and auditory motion processing in human cerebral cortex. Cereb. Cortex 10, 873-888.

Llinás, R., Urbano, F. J., Leznik, E., Ramirez, R. R., and van Marle, H. J. (2005). Rhythmic and dysrhythmic thalamocortical dynamics: GABA systems and the 
edge effect. Trends Neurosci. 28, 325-333.

Llinas, R., Ribary, U., Contreras, D., and Pedroarena, C. (1998). The neuronal basis for consciousness. Philos. Trans. R. Soc. Lond. B Biol. Sci. 353, 1841-1849.

Llinas, R., Ribary, U., Joliot, M., and Wang, X. (1994). "Content and context in temporal thalamocortical binding," in Temporal Coding in the Brain, eds G. Buzsaki, R. Llinasand, and W. Singer (Berlin: SpringerVerlag), 251-272.

Llinás, R. R., Ribary, U., Jeanmonod, D., Kronberg, E., and Mitra, P. P. (1999). Thalamocortical dysrhythmia: a neurological and neuropsychiatric syndrome characterized by magnetoencephalography. Proc. Natl. Acad. Sci. U.S.A. 96, 15222-15227.

Lockwood, A. H., Salvi, R. J., and Burkard, R. F. (2002). Tinnitus. N. Engl. J. Med. 347, 904-910.

Lorenz, I., Muller, N., Schlee, W., Hartmann, T., and Weisz, N. (2009). Loss of alpha power is related to increased gamma synchronizationA marker of reduced inhibition in tinnitus? Neurosci. Lett. 453, 225-228.

Masten, C. L., Eisenberger, N. I., Borofsky, L. A., Pfeifer, J. H., McNealy, K., Mazziotta, J. C., and Dapretto, M. (2009). Neural correlates of social exclusion during adolescence: understanding the distress of peer rejection. Soc. Cogn. Affect. Neurosci. 4, 143-157.

McKiernan, K. A., D’Angelo, B. R., Kaufman, J. N., and Binder, J. R. (2006). Interrupting the "stream of consciousness": an fMRI investigation. Neuroimage 29, 1185-1191.

Medford, N., and Critchley, H. D. (2010). Conjoint activity of anterior insular and anterior cingulate cortex: awareness and response. Brain Struct. Funct. 214, 535-549.

Menon, V., and Uddin, L. Q. (2010). Saliency, switching, attention and control: a network model of insula function. Brain Struct. Funct. 214, 655-667.

Melloni, L., Molina, C., Pena, M., Torres, D., Singer, W., and Rodriguez, E. (2007). Synchronization of neural activity across cortical areas correlates with conscious perception. J. Neurosci. 27, 2858-2865.

Mitchell, T. V., Morey, R. A., Inan, S., and Belger, A. (2005). Functional magnetic resonance imaging measure of automatic and controlled auditory processing. Neuroreport 16, 457-461.

Muhlau, M., Rauschecker, J. P., Oestreicher, E., Gaser, C., Rottinger, M., Wohlschlager, A. M., Simon, F., Etgen, T., Conrad, B., and Sander, D. (2006). Structural brain changes in tinnitus. Cereb. Cortex $16,1283-1288$.

Muhlnickel, W., Elbert, T., Taub, E., and Flor, H. (1998). Reorganization of auditory cortex in tinnitus. Proc. Natl. Acad. Sci. U.S.A. 95, 10340-10343.

Nicolas-Puel, C., Faulconbridge, R. L., Guitton, M., Puel, J. L., Mondain, M., and Uziel, A. (2002). Characteristics of tinnitus and etiology of associated hearing loss: a study of 123 patients. Int. Tinnitus J. 8, 37-44.

Norena, A., Cransac, H., and CheryCroze, S. (1999). Towards an objectification by classification of tinnitus. Clin. Neurophysiol. 110, 666-675.

Norena, A., Micheyl, C., CheryCroze, S., and Collet, L. (2002). Psychoacoustic characterization of the tinnitus spectrum: implications for the underlying mechanisms of tinnitus. Audiol. Neurootol. 7, 358-369.

Norena, A. J. (2011). An integrative model of tinnitus based on a central gain controlling neural sensitivity. Neurosci. Biobehav. Rev. 35, 1089-1109.

Norena, A. J., and Eggermont, J. J. (2003). Changes in spontaneous neural activity immediately after an acoustic trauma: implications for neural correlates of tinnitus. Hear. Res. 183, 137-153.

Norena, A. J., and Eggermont, J. J. (2006). Enriched acoustic environment after noise trauma abolishes neural signs of tinnitus. Neuroreport 17, 559-563.

Ohira, H., Nomura, M., Ichikawa, N., Isowa, T., Iidaka, T., Sato, A., Fukuyama, S., Nakajima, T., and Yamada, J. (2006). Association of neural and physiological responses during voluntary emotion suppression. Neuroimage 29, 721-733.

Ongur, D., and Price, J. L. (2000). The organization of networks within the orbital and medial prefrontal cortex of rats, monkeys and humans. Cereb. Cortex 10, 206-219.

Oppenheimer, S. (1993). The anatomy and physiology of cortical mechanisms of cardiac control. Stroke 24 (Suppl. 12), I3-I5.

Oppenheimer, S. M., Gelb, A. Girvin, J. P., and Hachinski, V. C. (1992). Cardiovascular effects of human insular cortex stimulation. Neurology 42, 1727-1732.

Phillips, M. L., Drevets, W. C. Rauch, S. L., and Lane, R. (2003) Neurobiology of emotion perception I: the neural basis of normal emotion perception. Biol. Psychiatry 54, 504-514.

Raichle, M. E., MacLeod, A. M., Snyder, A. Z., Powers, W. J., Gusnard, D. A., and Shulman, G. L. (2001). A default mode of brain function. Proc. Natl. Acad. Sci. U.S.A. 98, 676-682.

Rey, C., Reinecke, H. J., and Besser, R. (1984). Methyltin intoxication in six men; toxicologic and clinical aspects. Vet. Hum. Toxicol. 26, 121-122.

Ribary, U., Ioannides, A. A., Singh, K. D., Hasson, R., Bolton, J. P., Lado, F., Mogilner, A., and Llinas, R. (1991). Magnetic field tomography of coherent thalamocortical $40-\mathrm{Hz}$ oscillations in humans. Proc. Natl. Acad. Sci. U.S.A. 88, 11037-11041.

Rutkowski, R. G., and Weinberger, N M. (2005). Encoding of learned importance of sound by magnitude of representational area in primary auditory cortex. Proc. Natl. Acad. Sci. U.S.A. 102, 13664-13669.

Sadaghiani, S., Hesselmann, G., and Kleinschmidt, A. (2009) Distributed and antagonistic contributions of ongoing activity fluctuations to auditory stimulus detection. J. Neurosci. 29, 13410-13417.

Schlee, W., Weisz, N., Bertrand, O., Hartmann, T., and Elbert, T. (2008). Using auditory steady state responses to outline the functional connectivity in the tinnitus brain. PLoS One 3:e3720. doi: 10.1371/journal.pone.0003720

Schmitt, C., Patak, M., and KronerHerwig, B. (2000). Stress and the onset of sudden hearing loss and tinnitus. Int. Tinnitus J. 6, 41-49.

Seeley, W. W., Menon, V., Schatzberg, A. F., Keller, J., Glover, G. H., Kenna, H., Reiss, A. L., and Greicius, M. D. (2007). Dissociable intrinsic connectivity networks for salience processing and executive control. J. Neurosci. 27, 2349-2356.

Shargorodsky, J., Curhan, S. G. Curhan, G. C., and Eavey, R. (2010). Change in prevalence of hearing loss in US adolescents. JAMA 304 772-778.

Shulman, A. (1995). A final common pathway for tinnitus - the medial temporal lobe system. Int. Tinnitus J. 1, 115-126.

Steriade, M. (2006). Grouping of brain rhythms in corticothalamic systems. Neuroscience 137, 1087-1106.
Tiitinen, H., Sinkkonen, J., Reinikainen, K., Alho, K., Lavikainen, J., and Naatanen, R. (1993). Selective attention enhances the auditory 40$\mathrm{Hz}$ transient response in humans. Nature 364, 59-60.

van der Loo, E., Congedo, M., Vanneste, S., Van de Heyning, P., and De Ridder, D. (2011). Insular lateralization in tinnitus distress. Auton. Neurosci. 165, 191-194.

van der Loo, E., Gais, S., Congedo, M., Vanneste, S., Plazier, M. Menovsky, T., Van de Heyning, P., and De Ridder, D. (2009). Tinnitus intensity dependent gamma oscillations of the contralateral auditory cortex. PLoS One 4:e7396. doi: 10.1371/journal.pone.0007396

Vanneste, S., Plazier, M., van der Loo, E., Van de Heyning, P., and De Ridder, D. (2010a). The differences in brain activity between narrow band noise and pure tone tinnitus. PLoS One 5:e13618. doi: 10.1371/journal.pone.0013618

Vanneste, S., Plazier, M., der Loo, E., de Heyning, P. V., Congedo, M., and De Ridder, D. (2010b). The neural correlates of tinnitusrelated distress. Neuroimage 52, 470-480.

Vanneste, S., Van de Heyning, P., and De Ridder, D. (2011a). Contralateral parahippocampal gamma-band activity determines noise-like tinnitus laterality: a region of interest analysis. Neuroscience 199, 481-490.

Vanneste, S., Van de Heyning, P., and De Ridder, D. (2011b). The neural network of phantom sound changes over time: a comparison between recent-onset and chronic tinnitus patients. Eur. J. Neurosci. 34, 718-731.

Vanneste, S., Plazier, M., van der Loo, E., Van de Heyning, P., and De Ridder, D. (2011c). The difference between uni- and bilateral auditory phantom percept. Clin. Neurophysiol. 122, 578-587.

Voisin, J., Bidet-Caulet, A., Bertrand, O., and Fonlupt, P. (2006). Listening in silence activates auditory areas: a functional magnetic resonance imaging study. J. Neurosci. 26, 273-278.

Wang, J., Rao, H., Wetmore, G. S., Furlan, P. M., Korczykowski, M., Dinges, D. F., and Detre, J. A. (2005). Perfusion functional MRI reveals cerebral blood flow pattern under psychological stress. Proc. Natl. Acad. Sci. U.S.A. 102, 17804-17809.

Weisz, N., Moratti, S., Meinzer, M., Dohrmann, K., and Elbert, T. 
(2005). Tinnitus perception and distress is related to abnormal spontaneous brain activity as measured by magnetoencephalography. PLoS Med. 2:e153. doi: 10.1371/journal.pmed.0020153

Wheeler, R. E., Davidson, R. J., and Tomarken, A. J. (1993). Frontal brain asymmetry and emotional reactivity: a biological substrate of affective style. Psychophysiology 30, 82-89.
Yoo, S. S., Lee, C. U., and Choi, B. G. (2001). Human brain mapping of auditory imagery: event-related functional MRI study. Neuroreport 12, 3045-3049.

Zald, D. H., and Pardo, J. V. (2002). The neural correlates of aversive auditory stimulation. Neuroimage 16(3 Pt 1), 746-753.

Conflict of Interest Statement: The authors declare that the research was conducted in the absence of any commercial or financial relationships that could be construed as a potentia conflict of interest.

Received: 28 December 2011; accepted: 13 April 2012; published online: 08 May 2012

Citation: Vanneste S and De Ridder D (2012) The auditory and non-auditory brain areas involved in tinnitus. An emergent property of multiple parallel overlapping subnetworks. Front. Syst. Neurosci. 6:31. doi: 10.3389/fnsys. 2012.00031

Copyright (C) 2012 Vanneste and De Ridder. This is an open-access article distributed under the terms of the Creative Commons Attribution Non Commercial License, which permits non-commercial use, distribution, and reproduction in other forums, provided the original authors and source are credited. 